\title{
Comparison between Cationic Polymer and Lipid in Plasmidic DNA Delivery to the Cell Nucleus
}

\author{
Massimo Conese ${ }^{1,2, *}$ Alessandra Biffi ${ }^{1}$, Giorgia Dina ${ }^{3}$, Nicola Marziliano $^{4}$ and Antonello Villa ${ }^{5}$
}

\author{
${ }^{1}$ Institute for Experimental Treatment of Cystic Fibrosis, San Raffaele Scientific Institute, 20132 Milan, ${ }^{2}$ Department of \\ Biomedical Sciences, University of Foggia, 71100 Foggia, ${ }^{3}$ Neuropathology Unit, San Raffaele Scientific Institute, \\ 20132 Milan, ${ }^{4}$ Department of Animal Biology, University of Pavia, 27100 Pavia, ${ }^{5}$ Consorzio MIA, University \\ Milano/Bicocca 20052 Monza, Italy
}

\begin{abstract}
We investigated the cell association and intracellular pathway to the nucleus of complexes formed between DNA and cationic lipid DOTAP (lipoplexes) or cationic polymer polyethylenimine (polyplexes). Flow cytometry and confocal microscope analysis showed that lipoplexes presented higher affinity for cell membrane than polyplexes. Electron microscopy demonstrated that both types of complexes followed an endocytic pathway and were metabolized but did not enter the nucleus. However, by in situ PCR and FISH it was possible to show that the plasmid localized to the nuclei, indicating that DNA must be dissociated from the vectors to be delivered to the nucleus. Our results identify a different behaviour in the interaction of polyplexes and lipoplexes with the cell, indicating that a combination of advantageous properties of the two kinds of cationic molecules could further ameliorate efficiency of nonviral gene transfer vectors.
\end{abstract}

\section{INTRODUCTION}

Nonviral gene carriers offer the potential to safely and efficiently mediate the delivery of nucleic acid based therapeutics into targeted cells [1,2]. Viral vectors are able to mediate gene transfer with high efficiency and possibility of long-term gene expression. However, the acute immune response, immunogenicity [3], and insertional mutagenesis uncovered in recent gene therapy trials [4] have raised serious safety concerns about some common used viral vectors.

Nonviral vectors are typically based on cationic polymers or lipids, which upon self-assembly with plasmid DNA form complexes termed polyplexes or lipoplexes, respectively. Among the first nonviral vectors used [5, 6], cationic liposomes have displayed limited efficiency and high toxicity, due to high levels needed to overcome tissue and cellular constraints, coupled with their heterogeneity with respect to shape, size and composition [7]. The inherent limits of cationic liposomes have attracted towards the development of alternative cationic molecules, such as cationic polymers. Polyethylenimine (PEI) is perhaps the most active and most studied polymer for gene delivery. PEI is able to condense plasmid DNA (pDNA) and to deliver efficiently to the targeted cells in vitro and in vivo [1, 2]. PEI outperforms cationic lipids for in vitro gene delivery into primary airway epithelial cells [8] and for in vivo transfection when intravenous injection [9] or intratracheal instillation into the lungs [10] is performed. PEI is suitable also for gene delivery through aerosolisation of lipoplexes to treat genetically determined diseases, such as cystic fibrosis, or lung tumours [11]. Unfortunately, PEI is associated with

*Address correspondence to this author at the Department of Biomedical Sciences, University of Foggia, c/o Ospedali Riuniti, Viale L. Pinto 1, 71100 Foggia, Italy; E-mail: m.conese @unifg.it dose-dependent toxicity, which probably explains why it has not yet been used in human studies [12].

Once lipoplexes or polyplexes interact with negatively charged molecules such as glycosaminoglycans and are internalised, they follow and endocytic pathway, being localized at the level of endosomes [13, 14]. Escape from the endosome-lysosome compartment should occur and then either free pDNA or whole complexes must enter the nucleus in order pDNA to be transcribed. To explain and counteract PEI toxicity could be important to understand whether free DNA vehicled by PEI or the whole PEI/pDNA complexes enter the nucleus. This a controversial area since many evidences show that entire gene vector complexes are largely excluded from entry into non-mitotic nuclei [15-17], whereas others have found PEI/plasmid DNA complexes inside the nucleus, albeit at low levels [18, 19].

The main aim of our study was to study whether pDNA is delivered to the nucleus in form of free DNA or as entire complex and to compare PEI to the cationic lipid DOTAP (1,2-dioleoyloxy-3-(trimethylammonio)propane). Using endocytosis-interfering drugs, we have previously provided strong evidence that DOTAP lipoplex uptake proceeds only by clathrin-mediated endocytosis, while PEI polyplexes are taken up by two mechanisms, one involving caveolae and the other clathrin-coated pits [20]. We then sought to find whether there are any differences in delivering DNA to the nucleus between PEI and DOTAP.

\section{MATERIALS AND METHODOLOGY}

\section{Cell Culture}

A549 cells were from ATCC and were cultured in DMEM/Ham F12 (1:1) containing $10 \%$ foetal calf serum, $2 \mathrm{mM} \mathrm{L-glutamine,} 100 \mathrm{U} / \mathrm{ml}$ penicillin and 100 $\mu \mathrm{g} / \mathrm{ml}$ streptomycin. 


\section{Plasmids}

Plasmid pCLuc carries the $P$. pyralis luciferase coding region under the control of the cytomegalovirus (CMV) immediate-early enhancer/promoter region [10]. Plasmid pCMV-CFTR 4.6 carries the human Cystic Fibrosis Transmembrane Conductance Regulator gene $4.6 \mathrm{~kb}$ cDNA under the control of CMV enhancer/promoter [21]. Plasmid DNA preparation was performed by double $\mathrm{CsCl}$ gradient purification.

\section{Transfections}

Formation of DOTAP/DNA and PEI 25K/DNA complexes was carried out as previously described [9]. The following amounts of each vector were used per one microgram of DNA: $6 \mu \mathrm{l}$ of $1 \mathrm{mg} / \mathrm{ml}$ DOTAP solution (Boehringer Mannheim) or $0.3 \mu \mathrm{l}$ of $100 \mathrm{mM}$ PEI solution (Sigma). Cells were seeded onto 24-wells plate at 40,000/ well to obtain a $60-70 \%$ of confluency after an over-night incubation. The preformed complexes ( $2 \mu \mathrm{g}$ DNA) were added in $1 \mathrm{ml}$ of serum-containing medium. To evaluate luciferase expression, the plates were centrifuged for 10 minutes at $1,500 \mathrm{rpm}$ and the transgene activity was evaluated 24 hours later.

\section{Complexes Labelling}

Complexes were labelled by conjugation of DNA with FITC-poly-L-lysine (PLL; Sigma). FITC-PLL-containing complexes were prepared by pre-incubation of $3.6 \mu \mathrm{l}$ of 1 mM FITC-PLL with $2 \mu \mathrm{g}$ of DNA, followed by addition of either PEI or DOTAP. The presence of FITC-PLL did not give any decrease in the transfection efficiency. DNA was fluorescently labelled by inclusion of Texas Red-conjugated dUTP (ChromaTide Texas Red-12-dUTP, Molecular Probes, Eugene, OR). Incorporation of Texas Red-dUTP was performed by the Nick Translation System, according to manufacturer's instructions (GIBCO), by mixing labelled and unlabelled dUTP in a 1:5 w/w ratio. Separation between labelled DNA from non-incorporated nucleotides was performed with Sephadex G-50 Fine column (Amersham Pharmacia Biotech, Uppsala, Sweden).

\section{Cytofluorimetric Analysis}

Cells were incubated with either lipoplexes or polyplexes containing FITC-PLL for various time intervals at $37^{\circ} \mathrm{C}$, washed and analysed by fluorescence-activated cell sorting (FACS) with a FACScan apparatus (Becton-Dickinson, San Jose, CA). Ten thousand cells were used for each sample. The negative controls consisted of cells incubated with only the transfection medium. In order to obtain the specific number of fluorescent positive cells and the mean fluorescence, the values corresponding to the negative controls were subtracted from the values obtained from the transfected samples.

\section{Confocal Microscopy}

Cells were incubated with either lipoplexes or polyplexes containing Texas Red-conjugated DNA and FITC-PLL for 1-2 hours at $37^{\circ} \mathrm{C}$, washed, and fixed in $4 \%$ paraformaldehyde. Optical sections were obtained using the Bio-Rad MRC-1024 confocal microscope equipped with a Krypton/ Argon laser. In order to reduce bleed-through, double-label confocal images (both XY and $\mathrm{XZ}$ sections) were acquired sequentially. Digital images were processed using the program Laser Sharp 2000 (Bio-Rad).

\section{Electron Microscopy}

After the incubation with lipoplexes or polyplexes for various time intervals at $37^{\circ} \mathrm{C}$, cell culture monolayers were fixed in situ for 2 hours at room temperature with $4 \%$ paraformaldehyde/2\% glutaraldheyde in $120 \mathrm{mM}$ phosphate buffer, and then washed with the buffer. The monolayers were detached by scratching, suspended in the phosphate buffer, and centrifuged. The pellets obtained were washed extensively with $0,12 \mathrm{M}$ cacodylate buffer, postfixed with $2 \% \mathrm{OsO}_{4}{ }^{--}$in the same buffer, dehydrated in ethanol, and embedded in Epon. Thin sections, doubly stained with uranyl acetate and lead citrate, were examined in a Hitachi H-7000 electron microscope.

\section{In situ PCR and FISH on Isolated Nuclei}

Cells were incubated with DOTAP or PEI complexed to pCMV-CFTR 4.6 at $37^{\circ} \mathrm{C}$, detached and centrifuged at 1,500 $\mathrm{rpm}$ for 10 minutes. The pellet was incubated with $0.56 \%$ $\mathrm{KCl}$ for 30 minutes at $37^{\circ} \mathrm{C}$ and span down. Nuclei were fixed in methanol/acetic acid $(3: 1)$ at $4^{\circ} \mathrm{C}$ for 10 minutes, centrifuged, and finally resuspended in methanol/acetic acid. Fixed nuclei were deposited on glass coverslips and subjected to in situ PCR and FISH.

In situ PCR was performed following the method previously described [22]. The whole procedure was first validated on a reference gene (telomerase) and by using AMCA (aminomethylcoumarin acetate) as fluorescent dye in the FISH procedure. The amplification was performed by using specific primers for the hCFTR cDNA [21] and carried out onto a TC1000 system (Perkin-Elmer). The amplification mixture was as follows: $0.5 \mathrm{mM}$ of each primer, $4 \mathrm{mM} \mathrm{MgCl}_{2}, 0.2$ mM each of dATP, dGTP, dCTP, dTTP, 10 U of IS AmpliTaq DNA Polymerase and 1X Buffer II (all reagents were from Perkin-Elmer). The thermal profile of the reaction was: $72^{\circ} \mathrm{C}$ for 3 minutes, $60^{\circ} \mathrm{C}$ for 50 minutes for ten times. The reaction was stopped in $50 \mathrm{mM} \mathrm{NaCl}$ and $50 \mathrm{mM}$ EDTA and the amplicon fixed in 3:1 Methyl/Acetic for $10 \mathrm{~min}$ at $4{ }^{\circ} \mathrm{C}$.

After amplification, slides were processed for FISH. As probe was used pCMV-CFTR 4.6 labelled with biotin by the end labelling procedure (Boehringer Mannheim) following customer's instruction. Hybridisation mixture containing $50 \mathrm{ng}$ of biotinilated probe, $50 \%$ formamide, $2 \mathrm{x}$ SSC, $5 x$ Denhardt's solution and $500 \mathrm{mg} / \mathrm{ml}$ salmon sperm DNA, was applied onto slides for overnight hybridisation at $37^{\circ} \mathrm{C}$. Slides were washed three times in $50 \%$ formamide $/ 2 \mathrm{x}$ $\mathrm{SSC}$ at $42^{\circ} \mathrm{C}$ and three times in $2 \mathrm{x}$ SSC. Two rounds of amplification with fluorescein avidin, anti-avidin antibody (ONCOR) were applied to increase the hybridisation signal.

Cells were counterstained with Propidium Iodide $(0.3 \mathrm{mg} /$ $\mathrm{ml}$ ), mounted with an antifade (DABCO), and observed under a fluorescent microscope (Olympus, Provis), equipped with the appropriate filters set. Images were acquired by colour slide film (Scotchchrome 640T).

\section{RESULTS}

\section{Kinetics of Cell-Association of Lipoplexes and Polyplexes}

In initial studies we have compared the transfection kinetics of DOTAP/DNA complexes and PEI/DNA 
complexes. A549 cells were incubated with DOTAP and PEI complexed to pCLuc at different times at $37^{\circ} \mathrm{C}$, extensively washed, incubated in fresh medium up to 24 hours, and evaluated for luciferase levels. Fig. (1) shows that while DOTAP-containing complexes gave a quasi-maximal expression after only 15 minutes of incubation, PEI complexes reached significant levels after 2-6 hours of incubation.

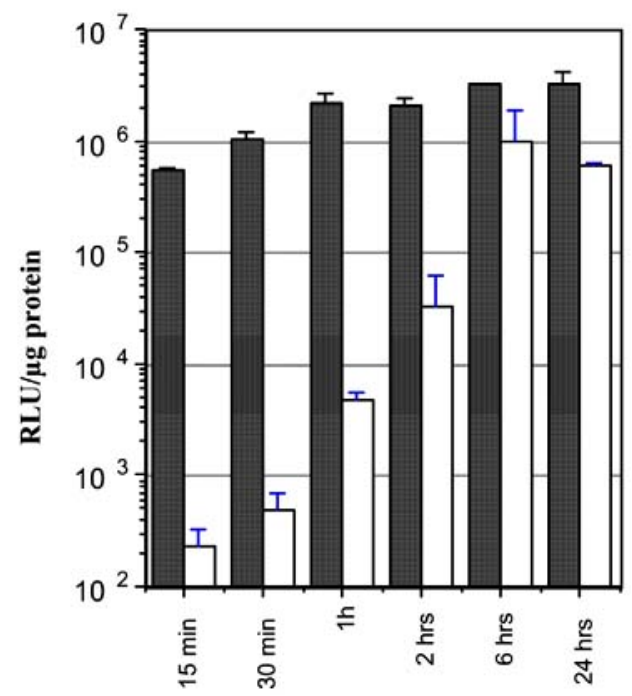

Fig. (1). Kinetics of transfection. A549 cells were incubated in the presence of cationic vector/pcLuc complexes, formed either with DOTAP (hatched columns) or PEI (white columns), for various time intervals at $37^{\circ} \mathrm{C}$, extensively washed, and then incubated up to 24 hours. Luciferase expression is expressed as Relative Light Units (RLU)/ $\mu \mathrm{g}$ protein. Data are expressed as the mean $\pm \mathrm{SD}$ of 3 experiments carried out in duplicate.

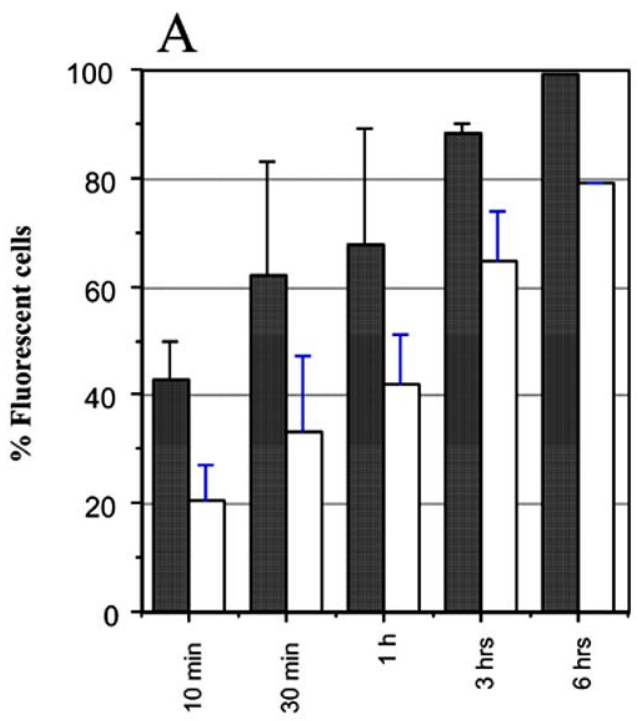

To study the kinetics of transfection in a more direct way, DNA was pre-condensed with fluoresceinated PLL prior to form the complexes. The pre-condensation of DNA with PLL did not decrease the luciferase expression mediated by DOTAP or PEI at any time length of incubation with the cells (data not shown). A549 cells were incubated with FITC-PLL-labelled complexes for different time intervals at $37^{\circ} \mathrm{C}$, washed off from the unbound complexes, and analysed by cytofluorimetry. After 10 minutes of incubation, DOTAP complexes associated to $40 \%$ cells, while PEI complexes only to $20 \%$ (Fig. 2A). Even if there was an increase in the number of fluorescent cells for both kind of complexes in a time dependent fashion, the difference in cell association efficiency between DOTAP and PEI complexes remained also at longer time intervals. The mean fluorescence, which describes the fluorescence on the whole cellular population, was 3-5 fold higher in lipoplexes-treated cells than in polyplexes ones (Fig. 2B). This difference maintained over time, indicating a greater number of fluoresceinated DOTAP complexes than PEI ones associated with the cells.

\section{Morphological Assessment of Cell-Associated Complexes}

Localization of FITC-labelled complexes was evaluated by confocal microscopy. In XY-sections, DOTAP complexes were found at the plasma membrane level in higher number than PEI complexes after 1 hour of incubation (Fig. 3A). In lipoplexes-treated cells, endocytic vesicles were noted under the plasma membrane that completely lacked in PEI-transfected cells (Fig. 3, compare $\mathbf{A}$ and E). The same pattern was found for Texas-Red-labelled DNA (Fig. 3B and $\mathbf{F}$ ), which exactly co-localized with FITC-PLL-labelled complexes (Fig. 3C and G), indicating that at this time point the complexes maintained their integrity. We also noted a light fluorescence signal apparently inside nuclei, a finding similar to that obtained by Godbey et al. [19]. Since this

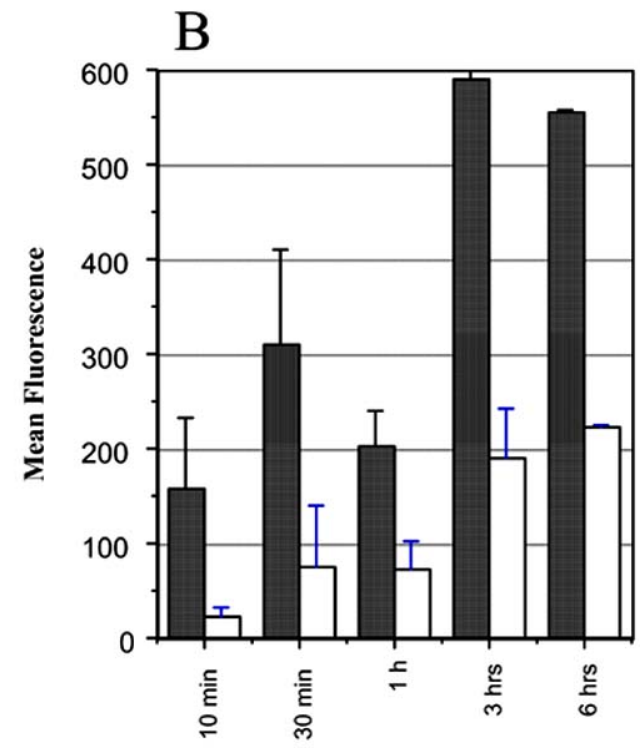

Fig. (2). Kinetics of cell association. A549 cells were incubated with complexes containing FITC-PLL for the indicated time intervals, washed, and evaluated by cytofluorimetry for the percentage of fluorescent cells (A) and the mean fluorescence (B). Data are expressed as the mean \pm SD of 3 experiments carried out in duplicate. DOTAP, hatched columns; PEI, white columns. 
weak signal could be localized either outside or into nuclei, we performed XZ-sections. However, no complexes were detected into the nuclei.
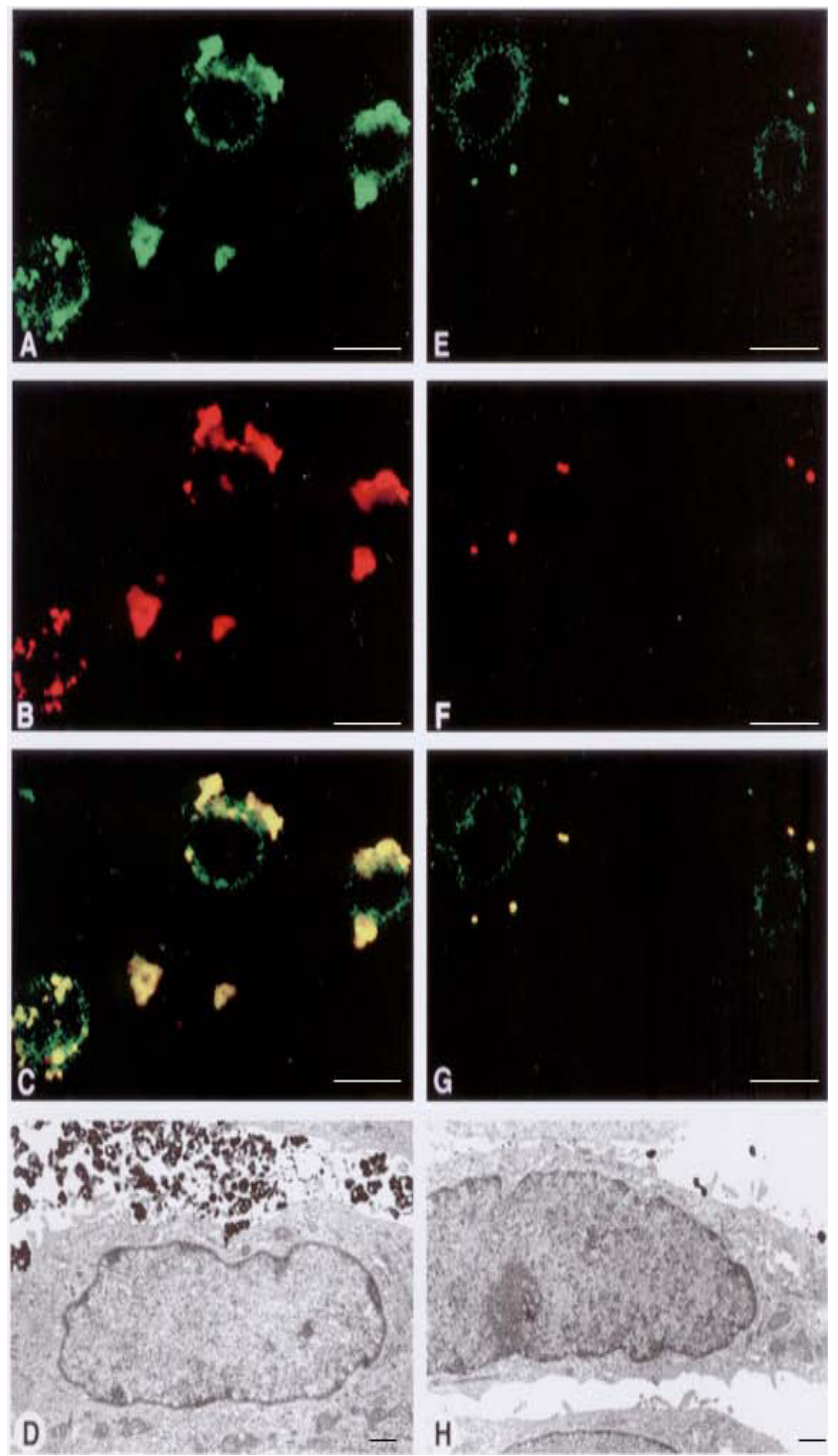

Fig. (3). Tracking of double-labelled complexes. A549 cells were incubated with lipoplexes $(\mathbf{A}, \mathbf{B}, \mathbf{C}$, and $\mathbf{D})$ or polyplexes $(\mathbf{E}, \mathbf{F}, \mathbf{G}$, and $\mathbf{H}$ ) containing FITC-PLL and Texas Red-conjugated DNA for 1 hour at $37^{\circ} \mathrm{C}$, washed, and evaluated at confocal microscopy. XYsections are shown. FITC and Texas Red signals co-localized (C and G). For electron microscopy, unlabelled lipoplexes (D) or polyplexes $(\mathrm{H})$ were incubated with cells for 2 hours at $37^{\circ} \mathrm{C}$, washed, and processed as described in Materials and Methods. Original magnification in A-C and E-G: X 60. Scale bar: $20 \mu \mathrm{m}$. Original magnification in D and H: X 500. Scale bar: $1 \mu \mathrm{m}$.

To determine precisely the localization of complexes, electron microscopy was performed on A549 cells treated with either lipoplexes or polyplexes. These experiments showed higher number of lipoplexes than polyplexes associated with the plasma membrane of A549 cells at 2 hours of incubation (Fig. 3D and $\mathbf{H}$ ). Under these experimental conditions, we never observed intranuclear localisation of complexes over 1,000 nuclear sections. Overall, these data point out that lipoplexes have a greater affinity for the cell membrane than polyplexes and that both types of complexes do not enter nuclei as such.

To study in more detail the uptake and the intra-cellular pathways of the complexes, an electron microscopy study was undertaken. At 2 hours of incubation, lipoplexes appeared as multilamellar structures inside endocytic vesicles. At this time, polyplexes particles were smaller and fewer than lipoplexes, but however located in endocytic pits. After 12 hours, lipoplexes appeared bigger and more electro-dense than at earlier time intervals and located in cytosolic vesicles, while polyplexes appeared as more dense irregular accumules encinclered by a membrane. At 24 hours, the cells presented electron-dense circular structures which were likely a metabolic product of the complexes. Fig. (4) shows a picture taken from the cells incubated for 24 hours and ricapitulates all these features of complexes inside the cells. Indeed, we found at 24 hours the same multi-lamellar structures observed at 2 and 12 hours for both lipoplexes and polyplexes, indicating that the endocytic process of complexes is continuous and asynchronous. Although these structures were also found very close to the nuclear membrane, we did not observe any electro-dense material in an intranuclear position (zero over 1,000 nuclear sections examined).

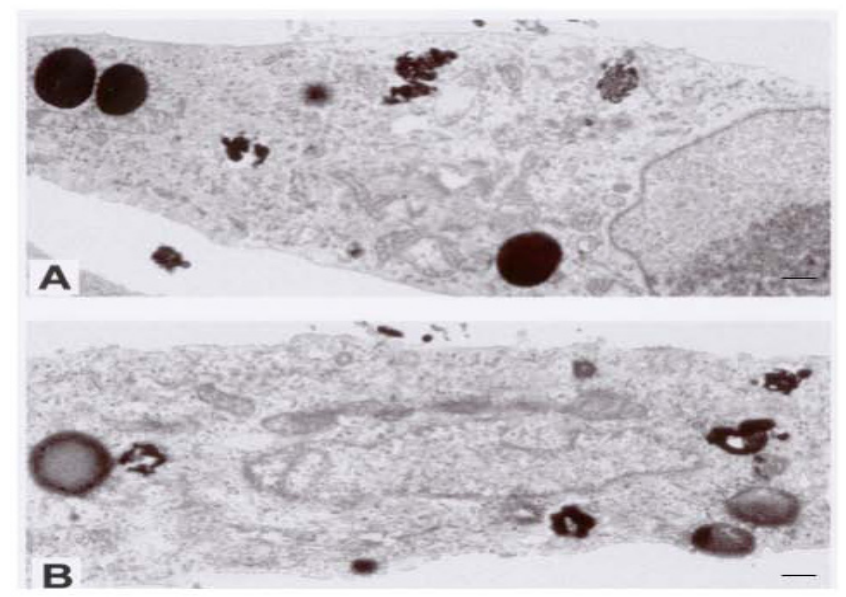

Fig. (4). The internalisation process is not synchronous. After 24 hours of incubation, lipoplexes (A) and polyplexes (B) appear in the same cell as multilamellar structures enveloped by a membrane, electron-dense accumules and rounded aggregates. Original magnification: X 500. Scale bar: $1 \mu \mathrm{m}$.

\section{Plasmid DNA Localisation in the Nucleus}

Previous data show clearly that entire DOTAP and PEI complexes did not gain access to the nucleus in this cell type. Since it was not possible to detect the naked plasmid by electron microscopy, we sought to reveal its nuclear localization by in situ PCR and hybridization. In control untransfected cells, specific signals were observed for a reference gene (telomerase) which was used for assessing the experimental procedure (Fig. 5A). In cells transfected with PEI/pCMV-CFTR complexes for 24 hours, we could detect different dots corresponding to the amplified plasmid (Fig. 5B). Since the PCR reaction was designed to amplify only plasmid-derived CFTR, CFTR specific signal was not detected in untransfected controls (not shown). Analysis 
of over 5,000 nuclei in each condition showed that, after 24 hours of incubation, cells presented specific nuclear fluorescence signal in $34 \%$ and $25 \%$ for DOTAP and PEI treatment, respectively (Table $\mathbf{1}$ ).
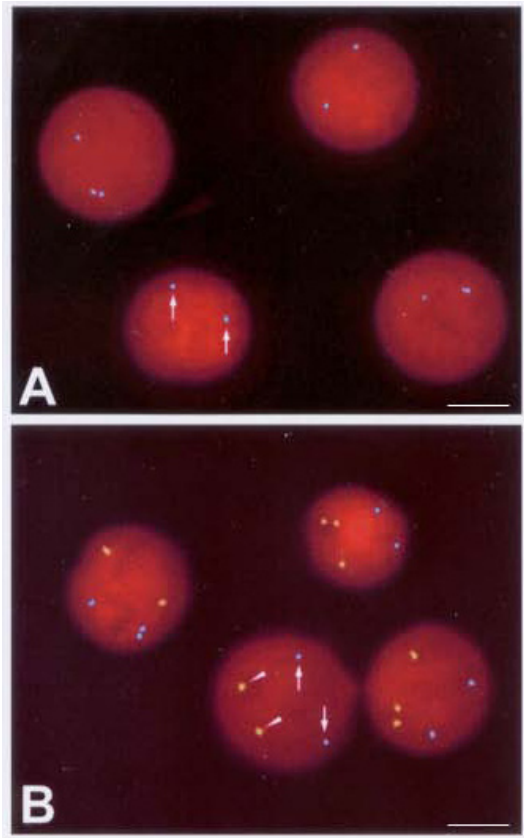

Fig. (5). In situ PCR and FISH on isolated nuclei. A549 cells were incubated with PEI/CMV-CFTR 4.6 for 24 hours at $37^{\circ} \mathrm{C}$. Nuclei were isolated and subjected to in situ PCR and hybridisation as described in Materials and Methodology. (A) Propidium iodidecounterstained nuclei of control cells (untransfected) showing telomerase AMCA hybridisation signals; the lower-left nucleus shows the typical chromatid duplication pattern (arrows). (B) Propidium iodide-counterstained nuclei of transfected cells showing hybridisation with boh AMCA-telomerase (arrows) and FITCCFTR (arrowheads). Untransfected cells did not show any specific signal for pCMV-CFTR 4.6. Original magnification: X 100. Scale bar: $10 \mu \mathrm{m}$

\section{DISCUSSION AND CONCLUSION}

Although cationic liposomes in toto and single components of them have already been using in Phase I clinical trials in cystic fibrosis [23] and other diseases (mainly cancer) [24], many aspects regarding the factors influencing their mechanism of action are still not well known. Our aims were to study the interaction of DNA/vector complexes with respiratory cells and to compare lipoplexes vs polyplexes. We used a cationic liposome, DOTAP, which has ben already used in human beings $[25,26]$ and a cationic polymer, PEI, which has been demonstrated to be a powerful vector to transfect different cell types in vivo [27].

The binding of lipoplexes and polyplexes to the cell surface is the result of a nonspecific ionic interaction between the positive charge of the complexes and the negative charge of the cell surface (reviewed in [28]). However, nothing is known about the kinetics of cell association and internalisation of complexes. It is well known that inhibitors of fluid-phase endocytosis such as chloroquine [29], low temperature [30], cholesterol depletion, chlorpromazine, potassium depletion [31], cytochalasin [32], etc. reduce transgene expression mediated by lipoplexes. However, endocytosis involves several sub-pathways. Wortmannin, a PI 3-kinase inhibitor, determined a variable inhibition of DOTAP:DOPE-mediated transfection depending on the cell-type, suggesting the involvement of PI 3-kinase dependent and independent lipoplexes uptake pathways [33]. Our data indicate that DOTAP lipoplexes associate and are endocytosed faster than PEI polyplexes (Figs. 2, 3). Using endocytosis-interfering drugs, we have previously provided strong evidence that DOTAP lipoplexes uptake proceeds only by clathrinmediated endocytosis, while PEI polyplexes are taken up by two mechanisms, one involving caveolae and the other clathrin-coated pits. Transfection by lipoplexes was entirely abolished by blocking clathrin-mediated endocytosis using potassium depletion of the cells [34], whereas inhibition of the caveolae pathway by pretreatment with genistein and filipin [35, 36] had no effect. By contrast, transfection mediated by polyplexes was completely blocked by genistein and filipin but was unaffected by inhibitors of clathrinmediated endocytosis [20]. Caveolae are slowly internalised and this might explain why DOTAP lipoplexes are endocytosed faster than PEI polyplexes. That PEI polyplexes uptake is mediated via both clathrin- and caveolae-mediated pathways and that only the DNA imported via caveolar uptake is expressed was confirmed by another recent study [37].

Table 1. Quantitative Analysis of Nuclear Localized-Plasmid by In Situ PCR and FISH

\begin{tabular}{|c|c|c|c|c|}
\hline \multirow[t]{2}{*}{ Nuclear Spots } & \multicolumn{2}{|c|}{ DOTAP } & \multicolumn{2}{|c|}{ PEI } \\
\hline & Nuclei & $\%$ & Nuclei & $\%$ \\
\hline 0 & 3700 & 65 & 3992 & 75 \\
\hline 2 & 1194 & 21 & 825 & 15.3 \\
\hline 4 & 198 & 3 & 74 & 1.4 \\
\hline
\end{tabular}

A549 cells were incubated with either DOTAP/DNA or PEI/DNA complexes for 24 hours at $37^{\circ} \mathrm{C}$. Nuclei were isolated and subjected to in situ PCR and hybridisation as described in Materials and Methodology. Epifluorescence microscopy revealed nuclei with 0, 2, 3, or 4 fluorescent spots. Percentages were calculated on the total number of evaluated nuclei which were 5,689 and 5,322 for DOTAP and PEI, respectively. 
Electron microscopy showed that complexes appeared to be vehicled through membrane enveloped vesicles (Fig. 5). It is likely that the lipidic or polymeric intracellular accumules seen at 24 hours do not represent anymore whole lipoplexes or polyplexes but only metabolized lipids or polymers. Nevertheless, escape of nonviral complexes from the endosomal compartment is essential for efficient transfection. A partial rupture of the endosomal membrane in case of lipoplexes, or their complete rupture in case of polyplexes has been proposed to be instrumental in the DNA release. With the former system, (transient?) endosomal membrane destabilization is thought to result from the intermingling of lamellar phase-perturbing lipids, including inverted hexagonal phase $\mathrm{H}_{\mathrm{II}}$ preferring cationic lipids, with the endosomal membrane [29, 38, 39]. The presence of the negatively charged lipid phosphatidylserine may amplify this destabilizing nonbilayer membrane organization upon its interaction with the cationic lipid and simultaneously cause the competitive dissociation of the DNA from the lipoplex [40] and its subsequent release into the cytosol. Additional phospholipids, such as dioleoylphosphatidylethanolamine may play a supportive role in these events ('helper lipid'). As initially proposed by Behr and coworkers [41, 42], strong protonation in the endosomal compartment of polyplexes like those consisting of PEI, accompanied by a concomitant neutralizing influx of chloride ions followed by influx of water, may result in osmotic rupture of endosomes and hence cytosolic release of polyplexes. Direct proof of the "proton sponge-mediated escape" theory was provided by Sonawane et al. [43]. These distinct mechanisms could explain why entire polyplexes are often seen within the cellular cytosol, whereas cationic lipids (or lipoplexes) remain largely localized within (or associated with) the endo/lysosomal membranes. Previously, Bieber et al. reported that PEI polyplexes accumulate in the lysosomal compartment after cellular uptake [44]. However, they only observed co-localisation between a fraction of the polyplexes and lysosomal markers. A significant part of the polyplexes resided in vesicles that did not co-cololize with lysosomal markers. These results indicate the presence of polyplexes in other vesicles that could represent caveosomes. Akinc et al. tested the protone sponge hypothesis and concluded that PEI particles avoid going to the lysosomes, because their surrounding $\mathrm{pH}$ averages 6.1 [45]. Based on this and other studies $[37,46]$, it is likely that the vesicles in which we observe PEI complexes represent a combination of both acidified andosomes/lysosomes and neutral $\mathrm{pH}$ caveosomes.

Intracellular injection experiments have shown that PEI, but not cationic lipids, enhance transgene expression when complexes are injected in the cytoplasm and that cationic lipids, but not PEI, prevent transgene expression when complexes are injected in the nucleus [47]. These data suggest that cationic polymers can allow the entrance of coated DNA in the nucleus where it is most likely released by competitive interaction with genomic DNA [48]. We could not detect whole lipoplexes or polyplexes at the nuclear level by extensive confocal and electron microscopy investigation, but plasmid by in situ PCR and FISH. Our data are in agreement with those previously reported by
Rémy-Kristensen et al. [49], who observed the absence of free PEI/DNA complexes in the nucleus of L929 cells. Sporadic observations on nuclear localisation of entire gene transfer complexes have been made. Godbey et al. [19] have showed by confocal microscopy the presence of polyplexes containing FITC-PEI in the nuclei of EA.hy 926 cells derived from a fusion of the human cell line A549 with human umbilical vein endothelial cells. Labat-Moleur et al. [50] found by electron microscopy a few percentage of nuclei containing DOGS/DNA complexes at 24 hours in MRC5 cells. To reconcile the absence of observable complexes in A549 nuclei with the transfection results, we speculate that the entry of the complexes in A549 nuclei may be a short-lived event involving a small number of complexes. Alternatively, our data indicate that plasmid DNA uncoats from cationic vectors before entering the nucleus.

Nonviral approach to gene transfer is not as efficient as viral-mediated gene delivery [51]. Unlike viral-mediated gene delivery, a process made nucleotropic with the involvement of several viral proteins, nonviral-mediated gene delivery is understood to be largely a stochastic process. Association of lipoplexes and polyplexes with the cell plasma membrane is believed to be a non-specific association. Cell surface binding of nonviral complexes is mostly driven by electrostatic interactions between complexes and proteoglycans present on the cell surface [52]. However, proteoglycans are also involved in the initial attachment of viral particles to the cells [53]. After receptor interaction, both enveloped and nonenveloped viruses must deliver their genome across either the endosomal or plasma membrane for infection to proceed. Genome delivery occurs either by membrane fusion (in the case of enveloped viruses) or by pore formation or other means of permeabilizing the lipid bilayer (in the case of nonenveloped viruses). Recent studies point out that caveolar endocytosis is essential for viral entry, such as for simian virus 40 [54] or echovirus 1 [55]. Thus, it would be interesting to investigate by the tools used in this study (electron microscopy combined with nuclear PCR and FISH) whether also viral vectors used in gene therapy (i.e. adeno-associated virus and lentivirus) are endocytosed and trafficked to the nucleus via the caveolar pathway.

In summary, we have previously showed that only the caveolae-dependent route leads to effective transfection while the DNA contained in the polyplexes internalized by clathrin-mediated endocytosis could not be released and was taken with the complex into the lysosomal compartment to be degraded [20]. On the other hand, data presented in this paper indicate that lipoplexes associate with a high affinity to plasma membrane of respiratory cells, while polyplexes present lower efficient interaction with the plasma membrane than lipoplexes. The main interesting feature of PEI is that it acts as a 'proton sponge'; its nitrogen atoms become protonated within acidic compartments like endosomes and lysosomes, triggering osmotic swelling of these vesicles. Structure/function studies will be needed to understand which domains of DOTAP and PEI are relevant for each step in the interaction with the cell. These data would provide a rationale for developing new hybrid cationic lipid/polymer vectors. 
ABBREVIATIONS

\begin{tabular}{|c|c|c|}
\hline AMCA & $=$ & Aminomethylcoumarin acetate \\
\hline CFTR & $=$ & $\begin{array}{l}\text { Cystic fibrosis transmembrane con- } \\
\text { ducatence regulator }\end{array}$ \\
\hline CMV & $=$ & Cytomegalovirus \\
\hline DOTAP & $=$ & $\begin{array}{l}\text { 1,2-dioleoyloxy-3- } \\
\text { (trimethylammonio)propane }\end{array}$ \\
\hline FISH & $=$ & Fluorescent in situ hybridisation \\
\hline PEI & $=$ & Polyethylenimine \\
\hline PLL & $=$ & Poly-L-lysine \\
\hline RLU & $=$ & Relative light units \\
\hline
\end{tabular}

\section{ACKNOWLEDGEMENTS}

This study was supported by a grant for Cystic Fibrosis from the Italian Ministero della Sanità (L 548/93).

\section{REFERENCES}

[1] Pack DW, Hoffman AS, Pun S, Stayton PS. Design and development of polymers for gene delivery. Nat Rev Drug Discov 2005; 4 : 581-93.

[2] Gao X, Kim KS, Liu D. Nonviral gene delivery: what we know and what is next. Aaps J 2007; 9: E92-104.

[3] Thomas CE, Ehrhardt A, Kay MA. Progress and problems with the use of viral vectors for gene therapy. Nat Rev Genet 2003; 4: 34658.

[4] Cavazzana-Calvo M, Fischer A. Gene therapy for severe combined immunodeficiency: are we there yet? J Clin Invest 2007; 117: 1456-65.

[5] Felgner PL, Gadek TR, Holm M, et al. Lipofection: a highly efficient, lipid-mediated DNA-transfection procedure. Proc Natl Acad Sci USA 1987; 84: 7413-7.

[6] Bennett MJ, Aberle AM, Balasubramaniam RP, et al. Cationic lipid-mediated gene delivery to murine lung: correlation of lipid hydration with in vivo transfection activity. J Med Chem 1997; 40: 4069-78.

[7] Ma B, Zhang S, Jiang H, Zhao B, Lv H. Lipoplex morphologies and their influences on transfection efficiency in gene delivery. $\mathrm{J}$ Control Release 2007; 123: 184-94.

[8] Casotti V, Syren M-L, Dina G, et al. Gene transfer in differentiated primary rat tracheal epithelial cells by non-viral vectors. Adv Gene Mol Cell Ther 2008; 1: 186-91.

[9] Bragonzi A, Boletta A, Biffi A, et al. Comparison between cationic polymers and lipids in mediating systemic gene delivery to the lungs. Gene Ther 1999; 6: 1995-2004.

[10] Bragonzi A, Dina G, Villa A, et al. Biodistribution and transgene expression with nonviral cationic vector/DNA complexes in the lungs. Gene Ther 2000; 7: 1753-1760.

[11] Densmore CL. Advances in noninvasive pulmonary gene therapy. Curr Drug Deliv 2006; 3: 55-63.

[12] Chollet P, Favrot MC, Hurbin A, Coll JL. Side-effects of a systemic injection of linear polyethylenimine-DNA complexes. J Gene Med 2002; 4: 84-91.

[13] Hoekstra D, Rejman J, Wasungu L, Shi F, Zuhorn I. Gene delivery by cationic lipids: in and out of an endosome. Biochem Soc Trans 2007; 35: 68-71.

[14] Rejman J, Conese M, Hoekstra D. Gene transfer by means of lipo- and polyplexes: role of clathrin and caveolae-mediated endocytosis. J Liposome Res 2006; 16: 237-47.

[15] Huth S, Hoffman F, von Gersdorff $\mathrm{K}$, et al. Interaction of polyamine gene vectors with RNA leads to the dissociation of plasmid DNA-carrier complexes. J Gene Med 2006; 8: 1416-24.

[16] Itaka K, Harada A, Yamasaki Y, et al. In situ single cell observation by fluorescence resonance energy transfer reveals fast intracytoplasmic delivery and easy release of plasmid DNA complexed with linear polyethylenimine. J Gene Med 2004; 6: 76-84.

[17] Grosse S, Thevenot G, Monsigny M, Fajac I. Which mechanism for nuclear import of plasmid DNA complexed with polyethylenimine derivatives? J Gene Med 2006; 8: 845-51.
[18] Grosse S, Aron Y, Honore I, et al. Lactosylated polyethylenimine for gene transfer into airway epithelial cells: role of the sugar moiety in cell delivery and intracellular trafficking of the complexes. J Gene Med 2004; 6: 345-56.

[19] Godbey WT, Wu KK, Mikos AG. Tracking the intracellular path of poly(ethylenimine)/DNA complexes for gene delivery. Proc Natl Acad Sci USA 1999; 96: 5177-81.

[20] Rejman J, Bragonzi A, Conese M. Role of clathrin- and caveolaemediated endocytosis in gene transfer mediated by lipo- and polyplexes. Mol Ther 2005; 12: 468-74.

[21] Biffi A, Sersale G, Cassetti A, et al. Restoration of bacterial killing activity of human respiratory cystic fibrosis cells through cationic vector-mediated CFTR gene transfer. Hum Gene Ther 1999; 10: 1923-30.

[22] Marziliano N, Crovella S, Zuccotti M, Garagna S. Six-year-old archival chromosome preparations are still good biological reagents for repeated primed in situ labelling (rPRINS). Eur J Histochem 1998; 42: 151-5.

[23] Bragonzi A, Conese M. Non-viral approach toward gene therapy of cystic fibrosis lung disease. Curr Gene Ther 2002; 2: 295-305.

[24] Morille M, Passirani C, Vonarbourg A, Clavreul A, Benoit JP Progress in developing cationic vectors for non-viral systemic gene therapy against cancer. Biomaterials 2008; 29: 3477-96.

[25] Porteous DJ, Dorin JR, McLachlan G, et al. Evidence for safety and efficacy of DOTAP cationic liposome mediated CFTR gene transfer to the nasal epithelium of patients with cystic fibrosis. Gene Ther 1997; 4: 210-8.

[26] McLachlan G, Ho L-P, Davidson-Smith H, et al. Laboratory and clinical studies in support of cystic fibrosis gene therapy using pCMV-CFTR-DOTAP. Gene Ther 1996; 3: 1113-23.

[27] Di Gioia S, Conese M. Polyethylenimine-mediated gene delivery to the lung and therapeutic applications. Drug Des Dev Ther 2008; 2: 163-88.

[28] Khalil IA, Kogure K, Akita H, Harashima H. Uptake pathways and subsequent intracellular trafficking in nonviral gene delivery. Pharmacol Rev 2006; 58: 32-45.

[29] Farhood H, Serbina N, Huang L. The role of dioleoyl phosphatidylethanolamine in cationic liposome mediated gene transfer. Biochim Biophys Acta 1995; 1235: 289-95.

[30] Zabner J, Fasbender AJ, Moninger T, Poellinger KA, Welsh MJ. Cellular and molecular barriers to gene transfer by a cationic lipid. J Biol Chem 1995; 270: 18997-19007.

[31] Zuhorn IS, Kalicharan R, Hoekstra D. Lipoplex-mediated transfection of mammalian cells occurs through the cholesterol-dependent clathrin-mediated pathway of endocytosis. J Biol Chem 2002; 277: 18021-8.

[32] Hui SW, Langner M, Zhao YL, et al. The role of helper lipids in cationic liposome-mediated gene transfer. Biophys J 1996; 71: 590-9.

[33] Prasad TK, Rangaraj N, Rao NM. Quantitative aspects of endocytic activity in lipid-mediated transfections. FEBS Lett 2005; 579: 2635-42.

[34] Larkin JM, Brown MS, Goldstein JL, Anderson RG. Depletion of intracellular potassium arrests coated pit formation and receptormediated endocytosis in fibroblasts. Cell 1983; 33: 273-85.

[35] Schnitzer JE, Oh P, Pinney E, Allard J. Filipin-sensitive caveolaemediated transport in endothelium: reduced transcytosis, scavenger endocytosis, and capillary permeability of select macromolecules. J Cell Biol 1994; 127: 1217-32.

[36] Orlandi PA, Fishman PH. Filipin-dependent inhibition of cholera toxin: Evidence for toxin internalization and activation through caveolae-like domains. J Cell Biol 1998; 141: 905-15.

[37] van der Aa MA, Huth US, Hafele SY, et al. Cellular uptake of cationic polymer-DNA complexes via caveolae plays a pivotal role in gene transfection in COS-7 cells. Pharm Res 2007; 24: 1590-8.

[38] Koltover I, Salditt T, Radler JO, Safinya CR. An inverted hexagonal phase of cationic liposome-DNA complexes related to DNA release and delivery. Science 1998; 281: 78-81.

[39] Smisterova J, Wagenaar A, Stuart MC, et al. Molecular shape of the cationic lipid controls the structure of cationic lipid/dioleylphosphatidylethanolamine-DNA complexes and the efficiency of gene delivery. J Biol Chem 2001; 276: 47615-22.

[40] Xu Y, Szoka FC, Jr. Mechanism of DNA release from cationic liposome/DNA complexes used in cell transfection. Biochemistry 1996; 35: 5616-23. 
[41] Behr JP. The proton sponge: a trick to enter cells the viruses did not exploit. Chimia 1997; 51: 34-36.

[42] Boussif O, Lezoualc'h F, Zanta MA, et al. A versatile vector for gene and oligonucleotide transfer into cells in culture and in vivo: Polyethylenimine. Proc Natl Acad Sci USA 1995; 92: 7297-301.

[43] Sonawane ND, Szoka FC, Jr., Verkman AS. Chloride accumulation and swelling in endosomes enhances DNA transfer by polyamineDNA polyplexes. J Biol Chem 2003; 278: 44826-31.

[44] Bieber T, Meissner W, Kostin S, Niemann A, Elsasser HP. Intracellular route and transcriptional competence of polyethylenimine-DNA complexes. J Control Release 2002; 82: 441-54.

[45] Akinc A, Thomas M, Klibanov AM, Langer R. Exploring polyethylenimine-mediated DNA transfection and the proton sponge hypothesis. J Gene Med 2005; 7: 657-63.

[46] Huth S, Lausier J, Gersting SW, et al. Insights into the mechanism of magnetofection using PEI-based magnetofectins for gene transfer. J Gene Med 2004; 6: 923-36.

[47] Pollard H, Remy J-S, Loussouarn G, et al. Polyethylenimine but not cationic lipids promotes transgene delivery to the nucleus in mammalian cells. J Biol Chem 1998; 273: 7507-11.

[48] Remy J-S, Kichler A, Mordvinov V, Schuber F, Behr J-P. Targeted gene transfer into hepatoma cells with lipopolyamine-condensed DNA particles presenting galactose ligands: a stage toward artificial virus. Proc Natl Acad Sci USA 1995; 92: 1744-8.
[49] Remy-Kristensen A, Clamme JP, Vuilleumier C, Kuhry JG, Mely Y. Role of endocytosis in the transfection of L929 fibroblasts by polyethylenimine/DNA complexes. Biochim Biophys Acta 2001; 1514: 21-32.

[50] Labat-Moleur F, Steffan A-M, Brisson C, et al. An electron microscopy study into the mechanism of gene transfer with lipopolyamines. Gene Ther 1996; 3: 1010-7.

[51] Hama S, Akita H, Iida S, Mizuguchi H, Harashima H. Quantitative and mechanism-based investigation of post-nuclear delivery events between adenovirus and lipoplex. Nucleic Acids Res 2007; 35: 1533-43.

[52] Mislick KA, Baldeschwieler JD. Evidence for the role of proteoglycans in cation-mediated gene transfer. Proc Natl Acad Sci USA 1996; 93: 12349-54.

[53] Smith AE, Helenius A. How viruses enter animal cells. Science 2004; 304: 237-42.

[54] Pelkmans L, Kartenbeck J, Helenius A. Caveolar endocytosis of simian virus 40 reveals a new two-step vesicular-transport pathway to the ER. Nat Cell Biol 2001; 3: 473-83.

[55] Pietiainen V, Marjomaki V, Upla P, et al. Echovirus 1 endocytosis into caveosomes requires lipid rafts, dynamin II, and signaling events. Mol Biol Cell 2004; 15: 4911-25.

(C) Conese et al.; Licensee Bentham Open.

This is an open access article licensed under the terms of the Creative Commons Attribution Non-Commercial License (http://creativecommons.org/licenses/by-nc/3.0/) which permits unrestricted, non-commercial use, distribution and reproduction in any medium, provided the work is properly cited. 